\title{
Gasterópodos asociados al alga calcárea Halimeda opuntia (Udoteaceae) en Puerto Rico
}

\author{
Cedar I. García-Ríos ${ }^{1}$, Francisco J. Soto-Santiago ${ }^{2}$, Ricardo J. Colón-Rivera ${ }^{3}$ \& \\ Javier R. Medina-Hernández ${ }^{4}$ \\ 1. Programa de Biología Marina Costanera, Departamento de Biología, Universidad de Puerto Rico en Humacao, \\ Estación Postal CUH, 100 Carr 908, Humacao, Puerto Rico 00791-4300; cedar.uprh@gmail.com; \\ 2. franciscoj_soto@yahoo.com; \\ 3. arrecife.pr@gmail.com; \\ 4. jmedinapr@gmail.com
}

Received 01-X-2007. Corrected 30-VI-2008. Accepted 31-VII-2008.

\begin{abstract}
Gastropods associated with the calcareous alga Halimeda opuntia (Udoteaceae) in Puerto Rico. Gastropod communities associated with the calcareous green alga Halimeda opuntia are described and compared for two Puerto Rico coral reefs: one in the northeast with front tradewinds (windward zone) and one in the southwest (leeward zone). We analyzed the content of 21 lots (2432 g) of H. opuntia from leeward zone and 15 lots (2448 g) from the windward zone. In total we recovered 526 gastropods (prosobranchs) classified in 54 taxa. Thirteen species of gastropods represented $79.6 \%$ of the community. Differences between species richness and mean density values were not significant $(\mathrm{p}>0.100)$. Forty species were identified from leeward and thirty one (31) species from windward. Gastropod mean density was $13.9 / 100 \mathrm{~g}$ of algae in leeward and $7.6 / 100 \mathrm{~g}$ in windward. Significant differences were found for the Simpson Diversity Index (0.91 in the leeward and 0.82 in the windward community; $\mathrm{t}=3.44, \alpha=0.005$ ). We found no correlation between the weight of the algae and the frequency of the gastropods $\left(\mathrm{R}^{2}=0.0893\right.$ in leeward, 0.0249 windward and 0.0923 for both). The relationship between species composition in lots of windward and leeward study sites was explored via polar ordination and no segregation was found. When compared, the gastropod communities found in H. opuntia collected in coral reefs in Puerto Rico were more similar within them than any other communities found in other genera of seaweed and in other environments. Our results agree with the hypothesis that $H$. opuntia offers the interstitial community a protected microhabitat from water movement impact. $H$. opuntia creates more homogenous conditions than those of the habitat where we found the algae. Rev. Biol. Trop. 56 (4): 1665-1675. Epub 2008 December 12.
\end{abstract}

Key words: Mollusca, gastropod, Halimeda opuntia, coral reef, Puerto Rico, Caribbean.

Las comunidades de moluscos asociados a macroalgas marinas en El Caribe han sido descritas por Warmke y Almodóvar (1963) y García-Ríos (1983). Warmke y Almodóvar (1963) recolectaron 25 especies de algas en el suroeste de Puerto Rico. Encontraron 90 especies de moluscos de los cuales $99 \%$ de los individuos eran gasterópodos. Al comparar los moluscos encontrados en algas rojas, verdes y pardas concluyeron que existe una mayor cantidad y diversidad de éstos asociados a las algas rojas. Sin embargo, de acuerdo a los datos de esa misma publicación, Halimeda opuntia (Linnaeus) Lamouroux, un alga verde calcárea, presentó mayor riqueza de especies que cualquiera de las especies de algas rojas.

García-Ríos (1983) describió las comunidades de gasterópodos asociadas a macroalgas marinas, que se recolectaron en cuatro hábitats: costa rocosa, arrecife de coral, manglar expuesto al oleaje y canales de mangle. En ese trabajo se determinó que existe una mayor similitud entre las comunidades de gasterópodos que coexisten en el mismo hábitat, independientemente 
del taxon del alga; además, que la comunidad de gasterópodos asociados a $H$. opuntia, en cualquier hábitat tenía mayor diversidad de especies que las demás algas. Las muestras de $H$. opuntia recolectadas en el arrecife presentaron la mayor riqueza en gasterópodos (53 especies). Trabajos similares, realizados en el Pacífico Central, confirman la alta riqueza de gasterópodos asociados a algas del género Halimeda (Naim 1988, Minton 1990).

Tanto Warmke y Almodóvar (1963) como García-Ríos (1983), encontraron a la mayoría de los gasterópodos en todas las algas $\mathrm{y}$ en todos los hábitats. En algunos casos encontraron mayor cantidad de una especie de gasterópodo por un grupo de algas o un hábitat, pero esa asociación no es obligatoria. Solo dos especies de gasterópodos (del orden Ascoglossa) mostraron asociación con una especie de alga. El gasterópodo con dos valvas Berthelinia caribbea Edmunds, 1963 está asociado a Caulerpa verticillata J.Agardh (Warmke 1966). De forma similar, Ocynoe antillarum Mörch, 1863 se encuentra en asociación exclusiva con Caulerpa racemosa (Forsskal) J. Agardh (Warmke y Abbott 1961, Warmke y Almodóvar 1972).

H. opuntia puede cubrir un $10 \%$ de la superficie de un arrecife, su aporte a los sedimentos calcáreos supera la de los corales (Scoffin 1992). Esta alga tiene un talo calcáreo articulado muy ramificado. Le ofrece a la comunidad asociada sostén físico y resistencia al golpe de las olas (Lembi y Waaland 1988). Tanto Warmke y Almodóvar (1963) como García-Ríos (1983) le atribuyen a estos rasgos físicos la alta riqueza de especies que encontraron en ella. Los gasterópodos que se encuentran en las algas son, en su gran mayoría, menores a los $5 \mathrm{~mm}$ de longitud, por lo que se pueden refugiar entre los intersticios de las ramificaciones del alga.

Las poblaciones de gasterópodos asociados a algas son facilitadores del flujo de energía a niveles tróficos superiores. Muchos son microherbívoros que se alimentan de fragmentos del alga o de las epífitas que crecen sobre ella (Shacklock y Doyle 1983, Naim 1988).
Estos, a su vez, son parte de la dieta de muchos peces, donde destacan miembros de las familias Labridae y Pomacentridae. Otros peces, de las familias Scaridae y Acanthuridae, los ingieren en forma accidental, cuando ingieren el alga (Randall 1967).

H. opuntia presenta adaptaciones para evitar su consumo (Hay 1997). Entre éstas se señala su talo muy calcificado en arreglos compactos (Hay 1981), su bajo contenido calórico (Lembi y Waaland 1988) y la producción de compuestos químicos (diterpenoides) que repele a algunos herbívoros (Steneck 1988). En esta última categoría de defensa se mencionan dos metabolitos: halimedatetracetato y halimedatrial (Paul y Fenical 1983 y 1986). A pesar de esto, Randall (1967) evidencia el consumo de H. opuntia por 13 especies de peces que son abundantes en los arrecifes del Caribe.

El objetivo de este trabajo es comparar las comunidades de gasterópodos asociados a $H$. opuntia que se encuentran en arrecifes de coral en lugares geográficamente opuestos. Estas estaciones de colección difieren en: energía del oleaje, cantidad de materia orgánica en sedimentos y cercanía de manglares. Se desea corroborar si la alta diversidad de gasterópodos es particular del ambiente arrecifal más protegido del fuerte oleaje como el encontrado en el suroeste de Puerto Rico (sotavento) y estudiados por Warmke y Almodóvar (1963) y GarcíaRíos (1983), o si también se puede encontrar en los arrecifes más expuestos en el noreste de la Isla (barlovento) y separados de la costa.

\section{MATERIALES Y MÉTODOS}

La estación de sotavento está localizada en el Cayo Enrique, La Parguera (municipio de Lajas), localizado en el suroeste de Puerto Rico, alrededor de la latitud $17^{\circ} 57^{\prime} 25^{\prime \prime}$ norte y longitud $67^{\circ} 02^{\prime} 34^{\prime \prime}$ oeste. La estación de sotavento está a $1.5 \mathrm{~km}$ de la costa, donde existen extensos manglares de borde. Las muestras de H. opuntia se recolectaron en la plataforma arrecifal, en el biotopo de Porites porites (Pallas, 1766). La cresta del arrecife está cubierta por mangle rojo (Rhizophora mangle Linnaeus) y una pradera 
de hierbas marinas se extiende hacia la laguna arrecifal. H. opuntia se recolectó en áreas protegidas del fuerte oleaje, pero con flujo de agua continuo, a un metro de profundidad. Todas las algas recolectadas mostraban acumulación de detrito y sedimentos oscuros.

La estación de barlovento está localizada en Cayo Icacos, parte del arrecife La Cordillera (municipio de Fajardo), localizado al noreste de Puerto Rico, cercana a la latitud $18^{\circ} 23^{\prime} 28^{\prime \prime}$ norte y longitud $65^{\circ} 35^{\prime} 49^{\prime \prime}$ oeste. En contraste con la estación de sotavento, no existen manglares en las cercanías de la estación de barlovento. Está a $2 \mathrm{~km}$ de la costa, donde se encuentran playas de arena y acantilados rocosos. Las muestras de $H$. opuntia se recolectaron en un arrecife cercano al noroeste de Cayo Icacos. H. opuntia está presente en espacios entre corales que se levantan del fondo entre uno y dos metros. Las algas se recolectaron aproximadamente a un metro de profundidad. La orilla del Cayo Icacos presenta lajas de arenisca y playas de arenas de alto contenido calcáreo. Las algas se recolectaron en áreas con mayor exposición al oleaje que la estación anterior. Los sedimentos liberados de las algas al colocarlas en las bolsas de colección eran de color gris claro.

Los viajes de colección se hicieron entre septiembre a noviembre de 2003, abril y agosto de 2004. Las muestras fueron seleccionadas en forma no aleatoria y se escogieron los ejemplares de mayor tamaño. Varias algas en contacto eran recolectadas como una sola unidad. Todas las muestras de $H$. opuntia fueron tomadas en aguas claras. Los lotes de algas se recolectaron en bolsas plásticas para evitar pérdidas de organismos. Se tomó solamente la parte superior del alga, para no atrapar organismos asociados al sustrato. Las muestras se colocaron en hielo para su transporte al laboratorio, donde se congelaron inmediatamente, hasta el momento de su análisis.

Las muestras se descongelaron y se lavaron con agua dulce sobre una serie de cernidores de plástico con apertura de malla de: $0.32 ; 0.64$; $0.50 ; 1$ y $2 \mathrm{~mm}$. El material recuperado (fragmentos del alga, sedimentos e invertebrados) se examinó por tamaño usando microscopios de disección. Se separaron los gasterópodos que estaban vivos en el momento de la recolección del alga y se preservaron en alcohol al $70 \%$. Las algas fueron pesadas húmedas, después de extraer el exceso de agua con papel secante. Para determinar el peso mínimo de una muestra representativa de la comunidad, se determinó la curva de aumento de especies de gasterópodos con la acumulación de peso de la muestra para cada estación, según Brower et al. (1998). Se recolectaron un total de 15 lotes (2432 g) de $H$. opuntia en la estación de sotavento y 21 lotes (2448 g) en la de barlovento.

Para la clasificación se utilizaron las guías taxonómicas de Abbott (1974) y Warmke y Abbott (1961). Para la actualización de la nomenclatura se utilizó la lista de cotejo de Ortiz-Corps, (1985 y 1998). Algunas identificaciones se corroboraron comparando los ejemplares con organismos en la Colección Biológica de la Universidad de Puerto Rico en Humacao (CBUPRH), donde se depositó toda la colección.

Se analizó la relación entre el peso del alga y las comunidades asociadas (análisis de correlación). Se compararon las comunidades calculando la riqueza, la densidad y la diversidad de las especies (Diversidad de Simpson) según Brower et al. (1998). La densidad se calculó dividiendo cantidad de gasterópodos por 100 gramos del alga, igual a los trabajos previos. Para evaluar el grado de agregación en la distribución de los individuos en los lotes recolectados, se calculó la razón de varianza y promedio para las especies de mayor frecuencia, siendo la magnitud de los valores mayores de uno (1) indicador del grado de agregación, según Brower et al. (1998).

Para discutir la relación entre nuestros datos y los de trabajos anteriores, se usó el índice de similitud Bray Curtis, así como la técnica de ordenación polar (Gauch 1982) usando distancia objetiva (distancia Sorensen) según Wishard 1969. Antes de establecer la distancia entre las muestras, en el gráfico de ordenación, se realizó una normalización de las especies, calculando el porcentaje de la presencia de cada especie (frecuencia) en cada estación. 


\section{RESULTADOS}

En el total de 36 lotes se recuperaron 526 gasterópodos (prosobranquios). El 96.2\% de los individuos fueron identificados y clasificados en 54 taxones (Cuadro 1). La curva de especies nuevas con la acumulación de peso del alga alcanzó su punto de inflexión (una pendiente menor del 10\%) con la acumulación de $600 \mathrm{~g}$ en la estación de sotavento y $700 \mathrm{~g}$ en la de barlovento. Se considera este peso el mínimo para describir estas comunidades (Brower et al. 1998). Trece especies de gasterópodos representaron el $79.6 \%$ de la muestra de la comunidad (Cuadro 1).

No encontramos diferencias significativas en el número de especies diferentes en la estación de sotavento (40) y barlovento (31) ( $p=0.16$, prueba exacta de Fisher). Encontramos proporciones similares en la representación de familias taxonómicas, 28 para toda la muestra, 24 en la estación de sotavento y 20 en la de barlovento (Cuadro 2).

De las especies con frecuencia de 3 o más individuos (21 especies), solo 2 se encontraron asociadas a las algas en la región de barlovento y 13 en la de sotavento (Cuadro 2). Tampoco se encontraron diferencias significativas en los valores de densidad promedio (cantidad de individuos en $100 \mathrm{~g}$ de peso húmedo del alga) que fue de 13.9/100 g en la estación de sotavento y 7.6/100 $\mathrm{g}$ en la de barlovento $(\mathrm{p}=0178$, prueba t) (Cuadro 1 y 2).

La especie con mayor frecuencia fue Sinezona (Scisurella) confusa Rolán and Luque, 1994, siendo la de mayor frecuencia relativa en las muestras de las estaciones de barlovento $(36.4 \%)$ y la segunda en las de sotavento (15.6\%). Si se consideran las cinco especies con mayor frecuencia, $S$. confusa es la única especie de alta frecuencia relativa común en ambas estaciones. En la estación de sotavento Caecum regulare Carpenter, 1858 es la especie de mayor frecuencia (19.2\%), la tercera es Amphithalamus vallei Aguayo and Jaume, 1947 (10.3), seguida por Pachystremiscus ornatus (Olsson and McGinty, 1958) (7.1\%) y Albania auberiana (d'Orbigny, 1842) (6.5\%). En la estación de barlovento la segunda especie más frecuente es Tricolia adamsii (H. C. Lea, 1846) (12.3\%). De esta especie solo un ejemplar se informa para las estaciones de sotavento. En forma similar los ejemplares del género Parviturbiodes, con frecuencia de $8 \%$ en las estaciones de barlovento, no se encontró en las de sotavento. Las especies de la familia Vitrinellidae y Bittiolum varium (Pfeiffer, 1840) son las de frecuencia intermedia en ambas estaciones (Cuadro 2).

Los valores de diversidad para ambas comunidades resultaron relativamente altos

\section{CUADRO 1}

Comparación de las muestras de gasterópodos en Halimeda opuntia en arrecifes de coral localizados en estaciones de sotavento y barlovento en Puerto Rico

TABLE 1

Comparison of the gastropod samples in Halimeda opuntia from leeward and windward in Puerto Rico coral reefs

$\begin{array}{lccc} & \text { Sotavento } & \text { Barlovento } & \text { Total } \\ \text { Cantidad de lotes en muestra } & 15 & 21 & 36 \\ \text { Peso húmedo de muestra }(\mathrm{g}) & 2432 & 2448 & 4880 \\ \text { Total de gastrópodos en muestra } & 339 & 187 & 526 \\ \text { Densidad (ind/100g alga) } & 13.9 & 7.6 & 10.8 \\ \text { Especies diferentes } & 40 & 31 & 54 \\ \text { Especies con 1 solo individuo } & 18 & 14 & 21 \\ \text { Diversidad de Simpson } & 0.91 & 0.82 & 0.90 \\ \text { Índice de Shanon-Weaver } & 1.22 & 1.04 & 1.27\end{array}$


CUADRO 2

Frecuencia absoluta y relativa (\%) de las especies de gasterópodos asociados a Halimeda opuntia en arrecifes de coral en estaciones de sotavento (Cayo Enrique, La Parguera, Lajas) y barlovento (Cayo Icacos, Fajardo) en Puerto Rico

TABLE 2

Absolute and relative (\%) frequency of gastropod species associated with Halimeda opuntia in coral reefs stations in leeward (Cayo Enrique, La Parguera, Lajas) and windward (Cayo Icacos, Fajardo), Puerto Rico

\begin{tabular}{|c|c|c|c|c|c|c|}
\hline \multirow{2}{*}{ Familia } & \multirow{2}{*}{$\begin{array}{l}\text { Frecuencia } \\
\text { Especie }\end{array}$} & \multicolumn{2}{|c|}{ Sotavento } & \multicolumn{2}{|c|}{ Barlovento } & \multirow{2}{*}{ total } \\
\hline & & Absoluta & $\%$ & Absoluta & $\%$ & \\
\hline Scissurellidae & Sinezona confusa Rolán and Luque, 1994 & 53 & 15.6 & 68 & 36.4 & 121 \\
\hline Caecidae & Caecum regulare Carpenter, 1858 & 65 & 19.2 & 3 & 1.6 & 68 \\
\hline Anabathridae & $\begin{array}{l}\text { Amphithalamus vallei Aguayo and Jaume, } \\
1947\end{array}$ & 35 & 10.3 & 1 & 0.5 & 36 \\
\hline Vitrinellidae & $\begin{array}{l}\text { Pachystremiscus ornatus (Olsson and } \\
\text { McGinty, 1958) }\end{array}$ & 24 & 7.1 & 4 & 2.1 & 28 \\
\hline Cerithiidae & Bittiolum varium (Pfeiffer, 1840) & 18 & 5.3 & 9 & 4.8 & 27 \\
\hline Rissoidae & Alvania auberiana (d'Orbigny, 1842) & 22 & 6.5 & 2 & 1.1 & 24 \\
\hline Tricoliidae & Tricolia adamsi (Philippi, 1853) & 1 & 0.3 & 23 & 12.3 & 24 \\
\hline Vitrinellidae & sp. \#1 & 11 & 3.2 & 10 & 5.3 & 21 \\
\hline Rissoinidae & Schwartziella bryerea (Montagu, 1803) & 15 & 4.4 & 1 & 0.5 & 16 \\
\hline Vitrinellidae & Parviturboides sp. & 0 & 0.0 & 15 & 8.0 & 15 \\
\hline Cerithiidae & Cerithium litteratum (Born, 1778) & 13 & 3.8 & 1 & 0.5 & 14 \\
\hline Vitrinellidae & sp. \#2 & 9 & 2.7 & 5 & 2.7 & 14 \\
\hline Columbellidae & Zafrona pulchella (de Blainville, 1829) & 5 & 1.5 & 6 & 3.2 & 11 \\
\hline Turridae & Crassispira nigrescens (C. B. Adams, 1845) & 9 & 2.7 & 0 & 0.0 & 9 \\
\hline Rissoellidae & Rissoella caribaea Rehder, 1943 & 3 & 0.9 & 4 & 2.1 & 7 \\
\hline Trochidae & Tegula fasciata (Born, 1778) & 6 & 1.8 & 0 & 0.0 & 6 \\
\hline Liotiidae & Arene tricarinata (Stearns, 1872)? & 4 & 1.2 & 1 & 0.5 & 5 \\
\hline Cerithiidae & Cerithium atratum (Born, 1778) & 4 & 1.2 & 0 & 0.0 & 4 \\
\hline Pyramidellidae & Miralda sp. \#1 & 4 & 1.2 & 0 & 0.0 & 4 \\
\hline Vitrinellidae & Cyclostremiscus sp. & 3 & 0.9 & 0 & 0.0 & 3 \\
\hline Marginellidae & Hyalina pallida (Linné, 1758) & 3 & 0.9 & 0 & 0.0 & 3 \\
\hline Pyramidellidae & Odostomia laevigata (d'Orbigny, 1841) & 3 & 0.9 & 0 & 0.0 & 3 \\
\hline Cystiscidae & Gibberula lavalleeana d'Orbigny, 1842 & 2 & 0.6 & 0 & 0.0 & 2 \\
\hline Pyramidellidae & Turbonilla levis (C. B. Adams, 1850) & 2 & 0.6 & 0 & 0.0 & 2 \\
\hline Rissoinidae & Schwartziella fischeri (Desjardin, 1949) & 1 & 0.3 & 1 & 0.5 & 2 \\
\hline Pyramidellidae & Turbonilla interrupta (Totten, 1835) & 1 & 0.3 & 1 & 0.5 & 2 \\
\hline Fissurellidae & Diodora $\mathrm{sp}$. & 1 & 0.3 & 1 & 0.5 & 2 \\
\hline Tornidae & Cochliolepis parasitica Stimpson, 1858 & 0 & 0.0 & 2 & 1.1 & 2 \\
\hline Fissurellidae & Fissurella sp. & 0 & 0.0 & 2 & 1.1 & 2 \\
\hline Buccinidae & Pisania sp. & 0 & 0.0 & 2 & 1.1 & 2 \\
\hline Tricoliidae & Tricolia bella (M. Smith, 1937) & 0 & 0.0 & 2 & 1.1 & 2 \\
\hline Triphoridae & sp. & 0 & 0.0 & 2 & 1.1 & 2 \\
\hline Triphoridae & Iniforis turristhomae (Holten, 1802) & 0 & 0.0 & 2 & 1.1 & 2 \\
\hline Rissoidae & Alvania sp. & 1 & 0.3 & 0 & 0.0 & 1 \\
\hline Eulimidae & Oceanida graduata de Folin, 1871 & 1 & 0.3 & 0 & 0.0 & 1 \\
\hline
\end{tabular}


CUADRO 2 (Continuación)

Frecuencia absoluta y relativa (\%) de las especies de gasterópodos asociados a Halimeda opuntia en arrecifes de coral en estaciones de sotavento (Cayo Enrique, La Parguera, Lajas) y barlovento (Cayo Icacos, Fajardo) en Puerto Rico

TABLE 2 (Continued)

Absolute and relative (\%) frequency of gastropod species associated with Halimeda opuntia in coral reefs stations in leeward (Cayo Enrique, La Parguera, Lajas) and windward (Cayo Icacos, Fajardo), Puerto Rico

\begin{tabular}{|c|c|c|c|c|c|c|}
\hline \multirow{2}{*}{ Familia } & \multirow{2}{*}{$\begin{array}{l}\text { Frecuencia } \\
\text { Especie }\end{array}$} & \multicolumn{2}{|c|}{ Sotavento } & \multicolumn{2}{|c|}{ Barlovento } & \multirow{2}{*}{ total } \\
\hline & & Absoluta & $\%$ & Absoluta & $\%$ & \\
\hline Eulimidae & Melanella jamaicensis (C. B. Adams, 1845) & 1 & 0.3 & 0 & 0.0 & 1 \\
\hline Caecidae & Meioceras nitidum (Stimpson, 1851) & 1 & 0.3 & 0 & 0.0 & 1 \\
\hline Cerithiopsidae & Cerithiopsis $\mathrm{sp}$. & 1 & 0.3 & 0 & 0.0 & 1 \\
\hline Turridae & Crassispira fuscescens Reeve, 1843 & 1 & 0.3 & 0 & 0.0 & 1 \\
\hline Turridae & Drillia $\mathrm{sp}$. & 1 & 0.3 & 0 & 0.0 & 1 \\
\hline Fasciolariidae & Leucozonia ocellata (Gmelin, 1791) & 1 & 0.3 & 0 & 0.0 & 1 \\
\hline Marginellidae & sp. & 1 & 0.3 & 0 & 0.0 & 1 \\
\hline Modulidae & Modulus modulus (Linné, 1758) & 1 & 0.3 & 0 & 0.0 & 1 \\
\hline Skeneidae & $\begin{array}{l}\text { Parviturbo rehderi Pilsbry and McGinty, } \\
1945 ?\end{array}$ & 1 & 0.3 & 0 & 0.0 & 1 \\
\hline Rissoinidae & Rissoina cancellata (Philippi, 1847) & 1 & 0.3 & 0 & 0.0 & 1 \\
\hline Triphoridae & $\begin{array}{l}\text { Marshallora nigrocincta auct non C. B. } \\
\text { Adams, } 1839\end{array}$ & 1 & 0.3 & 0 & 0.0 & 1 \\
\hline Pyramidellidae & Turbonilla sp. & 1 & 0.3 & 0 & 0.0 & 1 \\
\hline Liotiidae & Arene cruentata (Mühlfeld, 1829) & 0 & 0.0 & 1 & 0.5 & 1 \\
\hline Cerithiopsidae & Horologica pupa (Dall and Simpson, 1901) & 0 & 0.0 & 1 & 0.5 & 1 \\
\hline Muricidae & Trachypollia nodulosa (C. B. Adams, 1845) & 0 & 0.0 & 1 & 0.5 & 1 \\
\hline Pyramidellidae & Eulima auricincta (Abbott, 1958) & 0 & 0.0 & 1 & 0.5 & 1 \\
\hline Fossaridae & Fossarus orbignyi Fischer, 1864 & 0 & 0.0 & 1 & 0.5 & 1 \\
\hline Pyramidellidae & Odostomia sp. & 0 & 0.0 & 1 & 0.5 & 1 \\
\hline \multirow[t]{2}{*}{ Costellariidae } & Vexillum albocinctum (C. B. Adams, 1845)? & 0 & 0.0 & 1 & 0.5 & 1 \\
\hline & no identificados & 8 & 2.4 & 12 & 6.4 & 20 \\
\hline Total & & 339 & & 187 & & 526 \\
\hline
\end{tabular}

(Cuadro 1). La diversidad de Simpson fue mayor para la comunidad de sotavento $\left(\mathrm{D}_{\mathrm{S}}=\right.$ $0.91)$ que para la de barlovento $\left(\mathrm{D}_{\mathrm{S}}=0.82\right)$. La prueba t para la comparación de los valores del índice de diversidad de Simpson mostró diferencias significativas $(\mathrm{t}=3.44)$ para $\alpha=0.05$, (ecuaciones en Brower et al. 1998).

No encontramos correlación entre el peso de la muestra del alga y la frecuencia de gasterópodos en ésta. Los valores de $\mathrm{R}^{2}$ fueron de 0.0893 para sotavento, 0.0249 para barlovento y 0.0923 para todas las muestras juntas (Fig. 1).

\section{DISCUSIÓN}

Las estaciones de estudio seleccionadas presentan dos situaciones ambientales contrastantes dentro de los parámetros que permiten el desarrollo de arrecifes en Puerto Rico. Existen situaciones de mayor impacto por el oleaje, pero observamos el crecimiento del talo de $H$. opuntia con el volumen para poder sostener las comunidades que aquí se describen. A pesar de una selección de estaciones buscando diferencias, no las encontramos en parámetros importantes para 


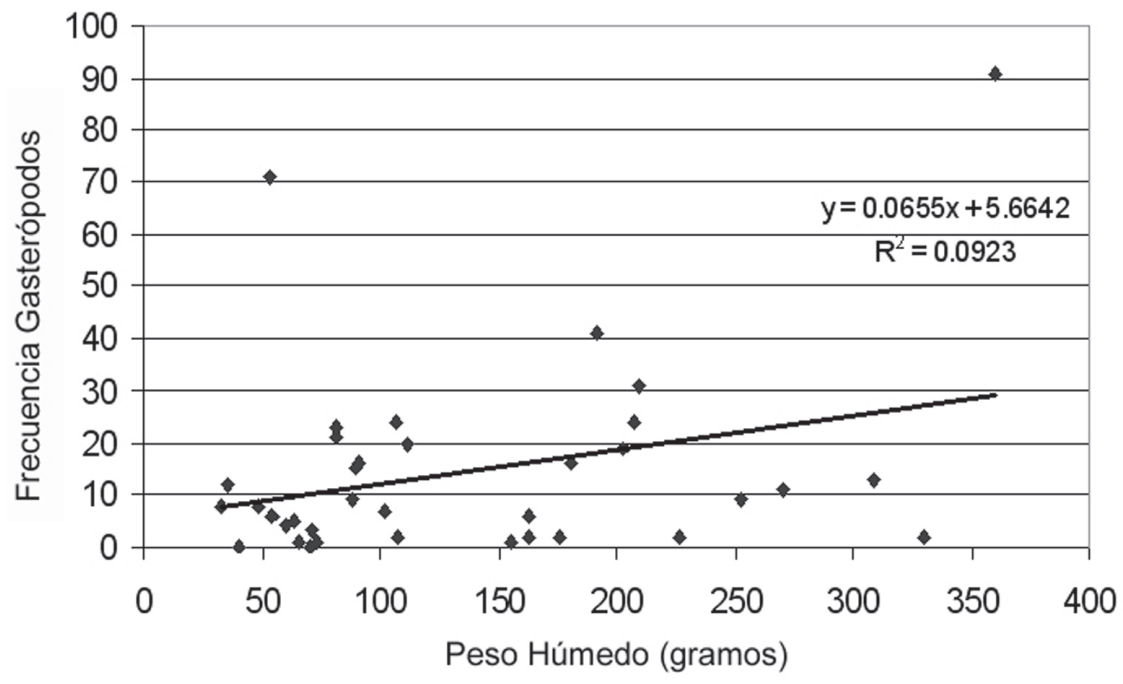

Fig. 1. Relación entre peso de las muestras y frecuencia absoluta de gasterópodos.

Fig. 1. Relationship between the sample weight and gastropod absolute frequency.

la descripción de la comunidad de gasterópodos asociada, como lo son el número de especies (riqueza de especies), su frecuencia y densidad. Estos resultados sostienen la hipótesis de que $H$. opuntia les ofrece a los organismos que viven en sus intersticios un microhábitat protegido del impacto del movimiento del agua, creando condiciones más homogéneas que las del hábitat donde crece el alga.

La ausencia de correlación entre el peso del alga y la frecuencia de gasterópodos asociados no corresponde a lo esperado, pues un alga de mayor tamaño debe ofrecer mayor superficie, recursos alimentarios y protección que un alga más pequeña. Tampoco corresponde con lo esperado, si consideramos que un alga de mayor tamaño debería reclutar más, como sugiere la teoría de islas (MacArthur 1972). Sin importar la localidad, el tamaño del alga no es un factor único para predecir parámetros de la comunidad asociada. Variables de naturaleza histórica, tales como la edad del alga y los disturbios que pueden haber afectado a las comunidades asociadas, son factores desconocidos y deben tener un impacto importante en estos resultados. Experimentos con sustratos artificiales, con características físicas similares a $H$. opuntia, podrían ayudar a comprender mejor la variabilidad encontrada. No obstante, se reconoce que el alga viva puede ser un agregador de larvas y juveniles significativamente superior que los sustratos artificiales que la imitan (Nugues y Szmant, 2006).

Los métodos de ordenación polar tampoco distinguen los lotes de sotavento de los de barlovento (Fig. 2). El porcentaje más alto de similitud de la comunidad entre las estaciones de sotavento fue de $29.1 \%$. Entre las estaciones de barlovento encontramos porcentajes de similitud tan altos como 59.6\%. Porcentajes relativamente bajos en similitud es característico de comunidades diversas, con baja dominancia y alta variabilidad en la frecuencia de especies entre los lotes de la misma estación. Las especies con mayor frecuencia en cada estación muestran patrones de agregación marcados (Cuadro 3).

La comparación de las comunidades de gasterópodos asociados a $H$. opuntia recolectadas en estaciones en barlovento y sotavento con las comunidades de gasterópodos en otras algas y otros hábitats confirma la similitud de 


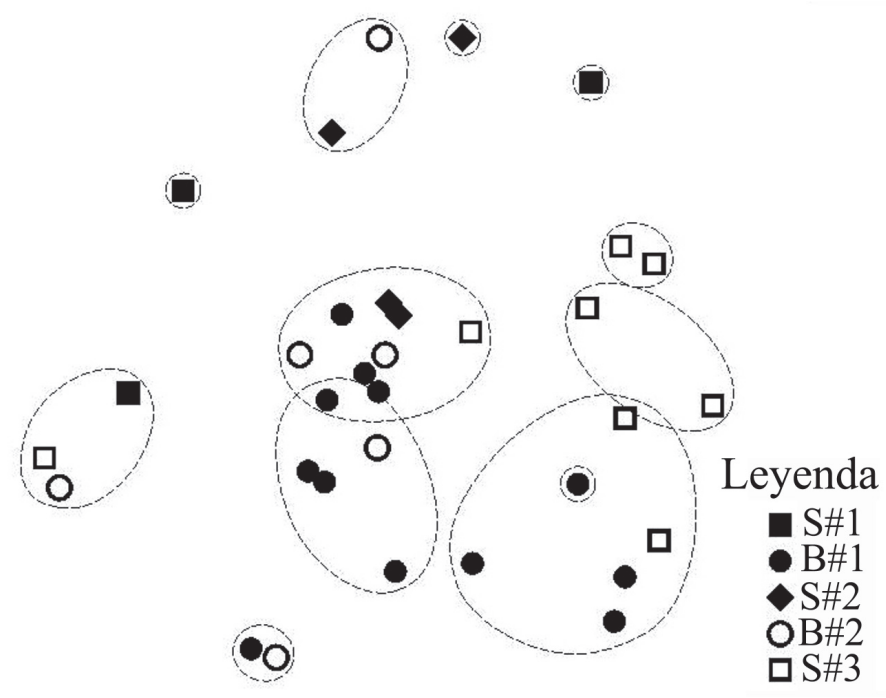

Fig. 2. Ordenación usando porcentaje de disimilaridad (distancia Sorensen) entre las comunidades de gasterópodos en 33 lotes de Halimeda opuntia (se omiten 3 que no tenían organismos), están agrupados por similitudes de 33\% o más.

Fig. 2. Ordination using percent of dissimilarity (Sorensen distance) between gastropod communities found in 33 lots of Halimeda opuntia (3 were omitted for lack of organisms), grouped by similarities of $33 \%$ or more. Windward $=\mathbf{\Delta}$; Leeward $=$

\section{CUADRO 3}

Razón varianza/promedio de la distribución de especies en los lotes de la estación de sotavento, barlovento y en combinación. Se consideran solo las especies con frecuencia mayor de 10

TABLE 3

Variance/mean ratio of the species distribution of samples in leeward, windward and both. Only species with frequency $>10$ are included

\begin{tabular}{lccc}
\multicolumn{1}{c}{ Especies } & Varianza/Promedio & Ambas \\
& Sotavento & Barlovento & 17.93 \\
Sinezona confusa & 27.69 & 8.72 & 4.61 \\
Caecum regulare & 4.27 & 36.97 \\
Amphithalamus vallei & 37.22 & 22.39 \\
Pachystremiscus ornatus & 25.50 & 3.06 \\
Bittiolum varium & 4.00 & 3.89 \\
Alvania auberiana & 4.12 & 2.05 & 2.44 \\
Tricolia adamsi & & 3.54 & 4.82 \\
Vitrinellidae sp. \#1 & 6.13 & & 2.40 \\
Schwartziella bryerea & 2.46 & 2.85 & 3.16 \\
Parviturboides ? & & & 1.43 \\
Cerithium litteratum & 1.36 & & 1.36 \\
Vitrinellidae sp. \#2 & & 5.05
\end{tabular}


las comunidades asociadas a $H$. opuntia en los arrecifes, independientemente de su localización. (Fig. 3). Para este análisis se agruparon los datos que corresponden a los lotes recolectados el mismo día en la misma estación. Esto reduce nuestras muestras de comunidades de gasterópodos en $H$. opuntia a cinco puntos, que son comparados con las 29 muestras de diferentes algas en diferentes ambientes de García Ríos (1983) y la de la comunidad de $H$. opuntia informada por Warmke y Almodóvar (1963) (Fig. 3). García-Ríos (1983) interpretó el eje vertical de este gráfico como indicador de energía de oleaje, siendo menor en los canales del manglar (abajo) y mayor en el arrecife y la costa rocosa (arriba). Nuestras muestras se localizan en la parte superior de esta ordenación, coincidiendo con la interpretación establecida. Las cinco muestras están muy cerca de las otras algas del arrecife y en un grupo de similitud mayor del $33 \%$ con las muestras de $H$. opuntia de arrecife de los dos trabajos anteriores.

En conclusión, las comunidades de gasterópodos asociados a $H$. opuntia creciendo en arrecifes de coral en zonas de barlovento y sotavento son similares en riqueza de especies y densidad promedio. Las dos comunidades descritas presentan valores de diversidad altos $\left(D_{S}>0.8\right)$, pero las de barlovento tienen menor diversidad. Las comunidades comparadas tienen mayor similitud entre sí, que con las encontradas en otras especies de algas y en otros ambientes. La alta similitud de estas

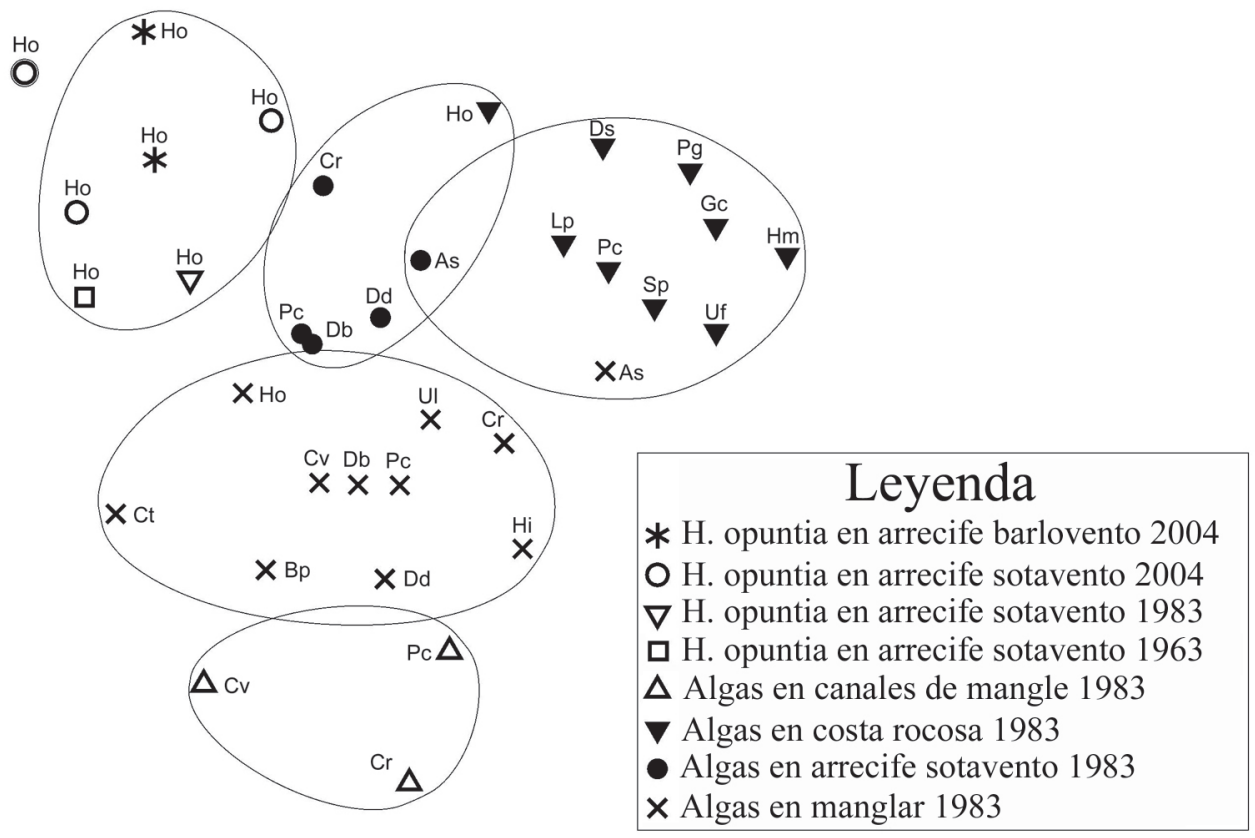

Fig. 3. Ordenación de las comunidades de gasterópodos asociadas a algas marinas en Puerto Rico. Se comparan los datos de Warmke y Almodóvar (1963), García-Ríos (1983) y este trabajo. Todos los datos se separan usando distancia Sorensen y se agrupan en similitudes de $30 \%$ o más. Se utilizan las siguientes abreviaturas para las algas: As, Acanthophora spicifera; Bp, Bryopsis pennata; Cr, Caulerpa racemosa; Ct, Caulerpa taxifolia; Cv, Caulerpa verticillata; Db, Dictyota bartayresii; Dd, Dictyota divaricata; Ds, Digenia simplex; Gc, Galaxaura cylindrica; Hi, Halimeda incrassata; Hm, Hypnea musciformis; Ho, Halimeda opuntia; Lp, Laurencia papillosa; Pc, Penicillus capitatus; Pg, Padina gymnospora; Sp, Sargassum polyceratium; Uf, Udotea flabellum y Ul, Ulva lactuca.

Fig. 3. Ordination of gastropod communities associated with seaweed in Puerto Rico. Data from Warmke and Almodóvar (1963), Garcia-Rios (1983) and this study. All data was arranged using Sorensen distance and grouped in similarities of $30 \%$ or more. 
comunidades se sostiene cuando comparamos los datos presentes con los de dos y cuatro décadas atrás.

\section{AGRADECIMIENTOS}

Se agradece a Rufo Vega Pagán y Migdalia Álvarez Ruiz por la ayuda en las recolectas y a Miguel Correa por su ayuda en el laboratorio. El microscopio electrónico de barrido, usado para la clasificación de las especies, fue facilitado por el programa Partnership for Research \& Education in Materials (PREM) de la Universidad de Puerto Rico en Humacao (NSF-DMR-0353730). Migdalia Álvarez Ruiz, Ariel Díaz y María Ramos fueron revisores de este manuscrito. Agradecemos al Fondo para la Investigación (FoPI) de la Universidad de Puerto Rico en Humacao y el programa Puerto Rico-Louis Stokes Alliance for Minority Participation (PR-LSAMP) por darnos acceso a equipo y materiales.

\section{RESUMEN}

Se describen y comparan las comunidades de gasterópodos asociados al alga verde calcárea $H$. opuntia. Se compararon estas comunidades en arrecifes coralinos de lugares geográfica y ambientalmente diferentes, uno en el noreste de Puerto Rico, frente a los vientos alisios (barlovento), el otro en el suroeste (sotavento). Se analizó el contenido de 15 lotes (2432 g) de $H$. opuntia recolectadas en la estación de sotavento y 21 lotes (2448 g) en la de barlovento. En los 36 lotes se recuperaron 526 gasterópodos (prosobranquios) que se clasificaron en 54 taxones. Trece especies de gasterópodos representaron el $79.6 \%$ de la muestra de la comunidad. No encontramos diferencias significativas en las densidades de gasterópodos ni en la riqueza de especies ( $p>0.100)$. La densidad promedio fue de 13.9/100 $\mathrm{g}$ alga en la estación de sotavento y 7.6/100 g en la de barlovento. Se identificaron 40 especies diferentes en las muestras de sotavento y 31 en las de barlovento. Solamente se encontraron diferencias significativas en los valores de diversidad (Simpson), de 0.91 para la comunidad de sotavento y de 0.82 en la de barlovento $(\mathrm{t}=3.44, \alpha=0.05)$. No encontramos correlación entre el peso de la muestra del alga y la frecuencia de gasterópodos en ésta, $\mathrm{R}^{2}=0.0893$ para sotavento, 0.0249 para barlovento y 0.0923 para datos combinados. Utilizando los métodos de ordenación polar se comprueba la similitud de las dos comunidades estudiadas. Estos resultados sostienen la hipótesis de que H. opuntia le ofrece a los organismos que viven en sus intersticios un microhábitat protegido del impacto del movimiento del agua, creando condiciones más homogéneas que las del hábitat donde encontramos creciendo el alga.

Palabras clave: gasterópodos, Halimeda opuntia, arrecife de coral, Puerto Rico, Caribe

\section{REFERENCIAS}

Abbott, R.T. 1974. American Seashells. Van Nostrand Reinhold, Nueva York, EEUU.

Brower J., J. Zar \& C. Von Ende. 1998. Field and Laboratory Methods for General Ecology. McGraw Hill, Massachusetts, EEUU.

García-Ríos, C.I. 1983. Comunidades de Gastrópodos Asociados a Macroalgas Marinas Comunes en el Suroeste de Puerto Rico. Tesis M.Sc. Departamento de Ciencias Marinas, Universidad de Puerto Rico, Mayagüez, Puerto Rico.

Gauch, H.G. 1982. Multivariate analysis in community ecology. Cambridge University, Nueva York, EEUU.

Hay, M.E. 1981. Spatial patterns of grazing intensity on a Caribbean barrier reef: herbivory and algae distribution. Aquat. Bot. 11: 97-109.

Hay, M.E. 1997. Calcified seaweeds on coral reefs: complex defenses, trophic relationships, and value as habitat. Proc. $8^{\text {th }}$ Int. Coral Reef Symp. 1: 713-718.

Lembi, C.A. \& J.R. Waaland. 1988. Algae and humans affairs. Cambridge University, Nueva York, EEUU.

MacArthur, R.H. 1972. Geographical Ecology. Harper and Row, Publishers, Nueva York, EEUU.

Minton, D. 1990. Microgastropods in Hawaiian Algae. Hawaiian shell news 38: 1-7.

Niam, O. 1988. Distributional pattern of mobile fauna associated with Halimeda on the Tiahura coral-reef complex (Morea, French Polynesia). Coral Reefs 6: 237-250.

Nugues, M.M. \& A.M. Szmant. 2006. Coral settlement onto Halimeda opuntia: a fatal attraction to an ephemeral substrate? Coral Reefs 25: 585-591.

Ortiz Corps, E.A.R. 1985. An annotated checklist of the recent marine Gastropoda (Mollusca) from Puerto Rico. Memorias del Sexto Simposio de la Fauna de Puerto Rico y el Caribe, pp. 1-220. University 
of Puerto Rico, Humacao University College, Department of Biology, Humacao, Puerto Rico.

Ortiz-Corps, E.A.R. 1998. The recent marine mollusca of Puerto Rico. Ph.D. Thesis, University of Puerto Rico, Mayagüez, Puerto Rico.

Paul, V.J. \& Fenical W. 1983. Isolation of Halimedatrial: chemical defense adaptation in the calcareous reefbuilding alga Halimeda. Science 221:747-749.

Paul, V.J. \& Fenical W. 1986. Chemical defense in tropical green algae, order Caulerpales. Mar. Ecol. Prog. Ser. 34:157-169.

Randall, J. E. 1967. Food habits of reef fishes of the West Indies. Stud. Trap. Oceanogr. 5: 665-847.

Shacklock, PF \& RW Doyle. 1983. Control of epiphytes in seaweed cultures using grazers. Aquaculture 31: 141-151.

Scoffin, T.P. 1992. Taphonomy of coral reefs: a review. Coral Reefs 11: 57-77.
Steneck, R.S. 1988. Herbivory on coral reefs: a synthesis. Proceeding of the $6^{\text {th }}$ International Coral Reef Symposium, Townsville, Australia, 1: 37-49.

Warmke, G.L. 1966. Two Species of the Bivalved Gastropod Berthelinia Found in Puerto Rico. Nautilus 79: 139141 .

Warmke, G.L. \& R. T. Abbott. 1961. Caribbean Seashells. Livingstone Publishing Company, Narberth, Pennsylvania, EEUU.

Warmke, G.L. \& L.R. Almodóvar. 1963. Some Associations of Marine Mollusks and Algae in Puerto Rico. Malacología 1: 163-177.

Warmke, G.L. \& L.R. Almodóvar. 1972. Observations on the Life Cicle and Regeneration in Oxynoe antillarum Morch, an Ascoglossan Opisthobranch from the Caribbean. Bull. Mar. Sci. 22: 67-74.

Wishart, D. 1969. An algorithm for hierarchical classifications. Biometrics 25:165-170. 
